Chapter 8

\title{
Glutamate Signaling in Human Cancers
}

\author{
Brian A. Wall, Seung-Shick Shin and Suzie Chen \\ Additional information is available at the end of the chapter \\ http://dx.doi.org/10.5772/55174
}

\section{Introduction}

G-proteincoupledreceptors(GPCRs)representaclassoftherapeutictargetsthathavebeenwidely exploitedfordrugdesignsand development.Metabotropicglutamatereceptors(mGluRs)belong to Class C GPCRs and are predominantly involved in maintaining cellular homeostasis in the central nervous system (CNS). The natural ligand of mGluRs, glutamate, interacts with receptor proteins leading to the activation of multiple signaling pathways. More recently, aberrant glutamate signaling has been shown to play a role in the transformation and maintenance of various cancer types, including melanoma. Glutamate secretion from these cells has been found to stimulate regulatory pathways that control tumor growth, proliferation and survival. In addition to synaptic transmissions, accumulating evidence suggesting other functional roles of glutamatergic signaling in human malignancies has presented intriguing possibilities to make mGluRs putative, novel targets for human cancer treatments. To this end, the aberrant expression of metabotropic glutamate receptor 1 (mGluR1) was found as the driving force in inducing melanomagenesis in transgenic mouse models. Since then, other subtypes of mGluRs have been implicated in the pathogenesis of various cancer types such as malignant gliomas and medulloblastomas. As such, increased efforts have been generated to elucidate the mechanisms by which mGluRs confer oncogenic potentials. This chapter summarizes our current knowledge on the participation of glutamate signaling in human cancers. Given that mGluRs are "druggable" members of the GPCR superfamily and their oncogenic implications in cancer, further understanding on anti-mGluR signaling pathways will be beneficial.

\section{Glutamate and cancer}

The amino acid L-glutamine is a precursor for peptide and protein synthesis, amino sugar synthesis, and nucleotide synthesis. The amide of glutamate, glutamine, is the most abundant 
free amino acid with a concentration ranging from 500-900 $\mu \mathrm{mol} / \mathrm{L}$. In the majority of cell types, the glutamine is rapidly converted to L-glutamate (we will refer to as glutamate), which is the most abundant intracellular amino acid. Glutamate is a key component in cellular metabolism contributing as a metabolic fuel for intermediates in energy producing pathways as well as an important component in the body's disposal of excess, or waste, nitrogen. Glutamate is a principal excitatory neurotransmitter in the mammalian central nervous system where it is involved in cognitive functions such as learning and memory. In an attempt to appease the insatiable needs that transformed cell types require to sustain their energy expenditure and biosynthetic requirements, tumor cells become major consumers of glutamine.

\subsection{The role of glutamate in normal and cancer cells}

Theuncontrolled proliferation thatrepresentsthehallmarkofneoplasticdiseasenotonlyinvolves deregulated cell proliferation but also requires adjustments in the energy metabolism that fuels the cells growth. Normal eukaryotic cells process the conversion of glucose to pyruvate via the metabolic pathway glycolysis in the cytosol, then to carbon dioxide in the mitochondria under aerobic conditions through the process of cellular respiration. In oxygen deprived environments, or hypoxic conditions, glycolysis is the preferred energy-generating pathway resulting in adecreasein theamountof pyruvateavailabletotheoxygen-consumingmitochondria.Ithasbeen observed that even in the presence of physiological relevant levels of oxygen cancer cells have the ability to reprogram their glucose metabolism allowing them to preferentially produce energy via the glycolysis while maintaining the same level of respiration. This "anaerobic respiration" is a process known as the Warburg Effect [1,2,3]. Although aerobic metabolism including oxidative phosphorylation is up to 15 times more efficient then anaerobic metabolism. However, the metabolism of cancer cells occurs at an increased glycolytic rate up to 200 times than those of their normal surrounding tissues. Cellular ATP production during such high rates of glycolytic flux can easily exceed the ATP produced via oxidative phosphorylation. This high consumption of glucose requires much more nicotinamide adenine (NAD+), which is consecutively regenerated from the conversion of pyruvate to lactate by increased expression of lactate dehydrogenase making glycolysis self-sufficient [8].

Glutamine serves as a major respiratory fuel of tumor cells and has been shown to be the absolute requirement over glucose for many malignant cell types [4]. The importance of glutamine in tumor cell metabolism is derived from its ability to satisfy the bioenergetic needs of tumor cells as well as providing intermediates for macromolecular synthesis [5]. During periods of rapid growth, the cellular demand for glutamine surpasses its supply. At this time glutamine becomes essential and it is this reason that glutamine has been designated a 'conditionally' essential amino acid. Traditionally, the role of glutamine has been viewed to function as storage for excess nitrogen and to traffic the nitrogen within, and between, various organs. Metabolically, glutamine is used directly for reactions that require its $\gamma$-nitrogen, such as nucleotide and hexosamine synthesis, or indirectly in reactions that utilize the $\alpha$-nitrogen (amino nitrogen) or its carbon skeleton for energy production and biosynthesis. These indirect reactions are necessary to generate the metabolic intermediates required for cell growth, which begin with the conversion of glutamine into glutamate. Being an abundant extracellular 
nutrient, glutamine is regularly converted into glutamate by phosphate-dependent glutaminase (GLS), an enzyme found within the inner mitochondrial membrane that has been shown to be overexpressed in both solid tumors and many tumor cell lines. In fact, the majority of glutamine consumption in cancer cells is owed to mitochondrial GLS activity. Consequentially, tumor cells have large intracellular pools of glutamate; through increased GLS activity, the abundantly available amino acid glutamine is converted to less abundant glutamate, there-by maintaining the cells' increasing demand for glutamate metabolism. This is the initial step in glutaminolysis, a series of biochemical reactions by which glutamine is degraded to glutamate, aspartate, pyruvate, alanine, citrate and carbon dioxide thereby providing metabolic intermediates to feed and maintain cells in which aerobic respiration is the biosynthetic hub.

Glycolysis and glutaminolysis both share the capacity to generate NADPH and the secretion of lactate (Figure 1). As mentioned previously, the initial step of glutaminolysis is the conversion of glutamine to glutamate, which is used, intracellularly, as a primary source of energy for proliferating cells. This rate-limiting step of glutamine metabolism is the deamidation reaction catalyzed by GLS. The products formed from this reaction, glutamate and ammonia, are released into the cytosolic compartment of the inner mitochondrial membrane. Glutamate is then further metabolized by conversion to $\alpha$-ketoglutarate via either oxidative deamination by glutamate dehydrogenase (GIDH) or alanine transaminase (ALT). The deamination reaction is localized to the mitochondrial matrix compartment and yields both $\alpha$-ketoglutarate and ammonium. The transamination can occur in either the cytosolic and mitochondrial compartments and produces $\alpha$-ketoglutarate and alanine. Alpha-ketoglutarate is a key intermediate in the citric acid cycle where it is metabolized to regenerate oxaloacetate (OAA) through the production of malate. Mitochondrial metabolism of $\alpha$-ketoglutarate into malate also generates NADPH via oxidation into pyruvate in the cytosol [6]. The NADPH produced is used by the rapidly metabolizing cell as a required electron donor in lipid synthesis and nucleotide metabolism as well as in antioxidant defense by maintaining glutathione (GSH) in its reduced state. GSH is the major thiol-containing endogenous antioxidant used by the cell to protect against various sources of oxidative stress. Maintaining a supply of GSH is critical for tumor cell survival since the elevated metabolic demand of the rapidly growing cell generates DNA-damaging byproducts that lead to oxidative stress [7].

Proliferating cancer cells consume vast amounts of glucose but only a small portion of which undergoes oxidative phosphorylation, even in the presence of sufficient oxygen [1]. It has been established that these rapidly dividing cells prefer to utilize glycolysis, which yields less net ATP molecules per glucose molecule. However, glycolysis can occur in hypoxic conditions that are usually associated with the highly necrotic regions of rapidly proliferating tumor masses. The end-product of glycolysis, pyruvate, can then enter the TCA cycle or is reduced into lactate by lactate dehydrogenase A, which is then excreted in the interstitial fluid. Within a tumor mass is a composition of genetically heterogeneous subpopulations of cancer cells that differ in their energy production pathways. It is possible that these subpopulations have the ability to function symbiotically within the tumor mass; one subpopulation of cells, consisting of hypoxic, glucose-dependent cells, secretes lactate as waste while the other subpopulation, located in a region that is more oxygenated, preferentially imports and utilizes the lactate 


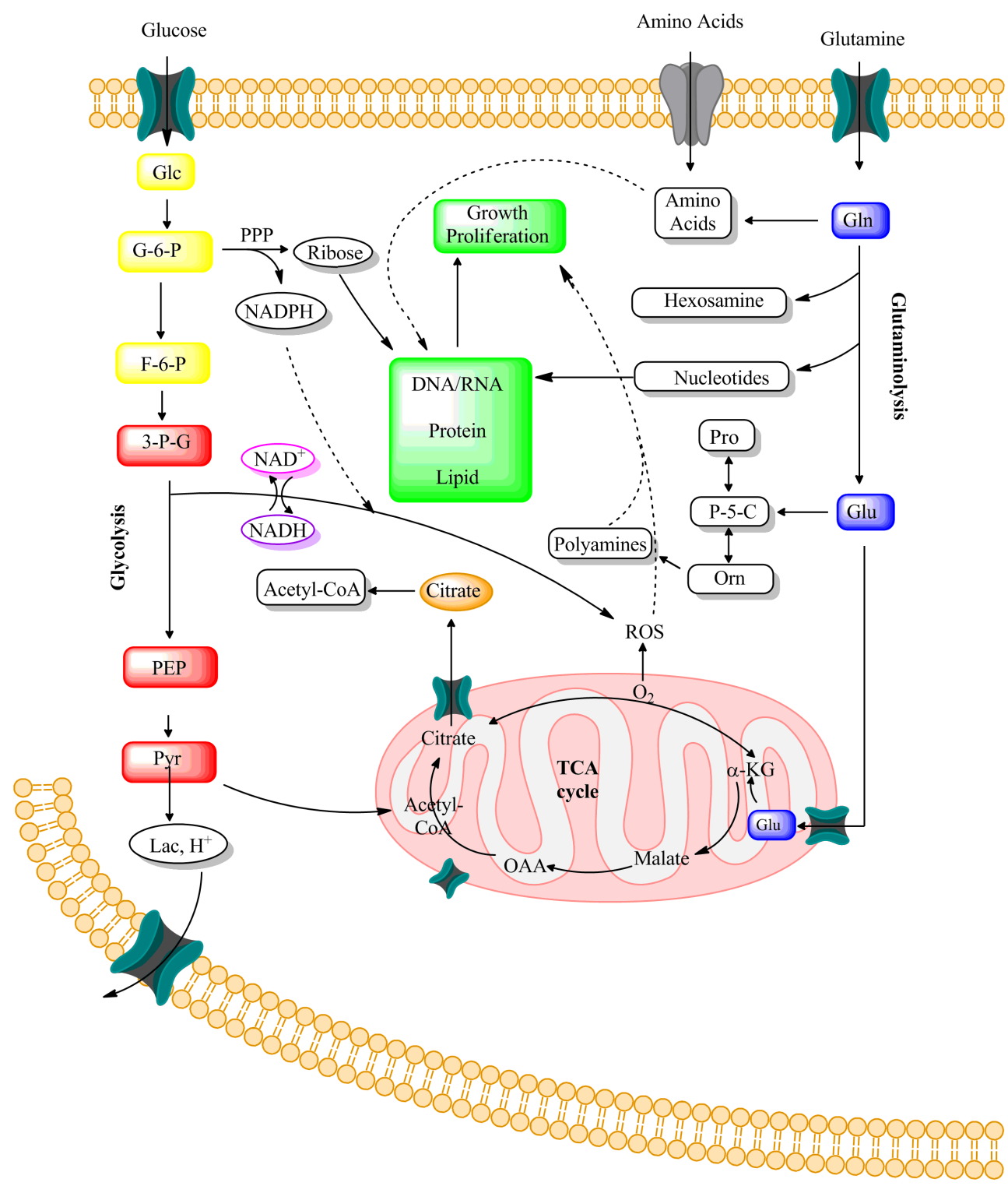

Figure 1. Metabolic pathways including glycolysis and glutaminolysis. Glycolysis produces NADPH and 5-carbon ribose via the pentose phosphate pathway (PPP) at glucose-6-phosphate (G-6-P) and detours toward lactate production (aerobic glycolysis) at pyruvate. The carbons of glucose are further diverted into various synthetic pathways to generate the precursors of hexosamines, amino acids and lipids. Mitochondria are fueled by the anapleurotic substrate, aketoglutarate $(\mathbf{a}-K G)$, generated from glutamate. Depending on the oxygen supply, a-ketoglutarate metabolizes through the tricarboxylic acid (TCA) cycle to malate and oxaloacetate (OAA) providing energy and a carbon resource for lipids. 
produced by the first subpopulation to be used in the citric acid cycle [8,9]. The idea of "metabolic symbiosis" that exists between hypoxic and aerobic cells in which lactate produced by hypoxic cells and taken up by aerobic cells to be used in oxidative support the bioenergetic needs of rapidly proliferating cancer cells within a solid tumor mass.

Cancer cells utilize TCA cycle intermediates to provide proliferating tumor cells with biosynthetic precursors (cataplerosis) as well as the production of ATP. The influx of metabolic intermediates in an attempt to maintain the mitochondrial TCA cycle (anaplerosis) is due to, in large part, glutaminolysis in which the first step is the conversion of glutamine to glutamate. Beyond its role in intermediary metabolism and biomass production, glutamate is also involved in influencing signal transduction pathways involved in promoting cellular proliferation, survival, and tumorigenesis. In the next section, we will review glutamate signaling and its role in the process of cellular transformation.

\section{Aberrant expression of G-protein coupled receptors in neoplastic transformation}

GPCRs are able to transduce extracellular signals into the cell via the activation of heterotrimeric G proteins. Malignant cells have been shown to appropriate the normal physiological functions of GPCR and utilize them to upregulate cell proliferation, invade surrounding tissue, increase blood supply, and evade cell death. In this section we will address the roles of GPCRs in tumor progression and metastasis.

\subsection{G-Protein coupled receptors}

Guanine nucleotide binding-protein coupled receptors (GPCRs; G-protein coupled receptors) comprise a large and diverse family of seven-pass membrane helix proteins found in most eukaryotic organisms. Members of the GPCR superfamily are diverse in their primary structure. The principal function of GPCRs is to transmit information about the extracellular environment via recognition of a variety of ligands including peptide and non-peptide hormones and neurotransmitters, chemokines, prostanoids and proteinases, biogenic amines, nucleosides, lipids, growth factors, odorant molecules and light to the interior of the cell by interacting with G-proteins. G-proteins are heterotrimeric subunits, $\mathrm{G}_{\alpha}, \mathrm{G}_{\beta}$ and $G_{\gamma}$, associated with the inner surface of the plasma membrane where they function as dimers. These G-proteins regulate the activity of small molecules that act as second messengers. Most of the human GPCRs can be found divided into five families based on phylogenetic criteria: Glutamate, Rhodopsin, Adhesion, Frizzled/Taste2 and Secretin (GRAFS classification system) [10]. The total number of known and verified human GPCRs consists of over 800 members. The Glutamate family consists of 22 human proteins categorized into ionotropic receptors (iGluRs) and metabotropic receptors (mGluRs). The iGluR family consists of $N$-methyl-D-aspartate receptors (NMDAR, $\alpha$-amino-3-hydroxy-5-methyl-4isoazolepropionic acid receptors (AMPAR), and kainite receptors (KAR). The mGluR family consists of eight different subtypes (mGluR1-8) [11]. The mGluRs are coupled to G- 
proteins, which distinguishes them from iGluRs, which contain ligand-gated ion-channels. The mGluRs are key receptors in the modulation of excitatory synaptic transmission in the central nervous system (CNS). The eight subtypes of mGluRs are further classified into three groups (I, II, III) based on sequence homology, signal transduction pathways, and agonist selectivity. mGluR1 and 5 belonging to subgroup I; mGluR2 and 3 belonging to subgroup II; and mGluRs 6, 7, and 8 belonging to subgroup III. The structures of mGluRs are composed of a heterotrimeric extracellular region, a seven- $\alpha$ helical transmembrane region and a cytoplasmic region. The extracellular region is further divided into the ligandbinding region (LBR) and the cysteine-rich region. The LBR has a sequence homologous with the extracellular regions of both iGluR and the $\gamma$-amino butyric acid (GABA) receptor $[12,13]$. Table 1 lists the expression of mGluRs in non-neuronal cells.

\begin{tabular}{ccc}
\hline Cell Type & GPCR & Reference \\
\hline Melanocytes & mGluR5 & Frati et al.,2000 \\
\hline Kerotinocytes & mGluR1,2 & Genever et al.,1999 \\
\hline Osteoclasts & mGluR8 & Morimoto et al.,2006 \\
\hline Pancreatic Islets/ $\beta$ cells & mGluR5,8 & Storto et al.,2006 \\
\hline Hepatocytes & mGluR5 & Tong et al.,2002 \\
\hline Myocytes & mGluR1,2,3,5 & Storto et al.,2000b \\
\hline Thymocytes & mGluR1,2,3,5 & Gill et al.,1999 \\
\hline Embryonic Stem Cells & mGluR4,5 & Storto et al.,2000a \\
\hline
\end{tabular}

Table 1. Expression of mGluRs in select non-neuronal cells

\subsection{GPCR signaling}

The two principal signal transduction pathways involving GPCRs are the cAMP signaling pathway and the phosphatidylinositol signaling pathway. Glutamate-stimulated activation causes a conformational change in the extracellular domains, which catalyze the exchange of a GTP for a GDP on the G $\alpha$ subunit of the intracellularly coupled G-protein. This exchange decreases the affinity of the $G \alpha$ subunit for the $G \beta \gamma$ monomer at which time they either dissociate or rearrange [14, 15]. The dissociated $G \alpha$ and $G \beta \gamma$ subunits interact with and modulate downstream signaling targets including adenylyl cyclases, phosphodiesterases, phosphlipases, tyrosine kinases, and ion channels.[16, 17]. Group I mGluRs are coupled to $\mathrm{G}_{\alpha q} / \mathrm{G}_{\alpha / 11}$ G-proteins that, upon glutamate-mediated activation, result in stimulation of phospholipase $\mathrm{C}$ beta $\left(\mathrm{PLC}_{\beta}\right)$. Activation of $\mathrm{PLC}_{\beta}$ causes hydrolytic cleavage of phosphatidylinositol-4, 5-diphosphate $\left(\mathrm{PIP}_{2}\right)$ resulting in the formation of diacyl glycerol (DAG) and inositol 1, 4, 5-triphosphate $\left(\mathrm{IP}_{3}\right)$. DAG remains bound to the membrane while IP3 is released as a soluble structure into the cytosol. Release of these secondary messengers results in increased calcium release from the endoplasmic reticulum and activation of protein kinase $\mathrm{C}$ 
(PKC) which phosphorylates other molecules, leading to altered cellular activity (Figure 2). Group II and III mGluRs are coupled to $G_{\alpha i / o}$ G-proteins that, upon activation prevent the formation of cyclic adenosine monophosphate (cAMP). Upon activation, group II mGluRs couple to $G_{\alpha i / o}$ subunit. $G_{\alpha i / o}$ G-proteins can inhibit adenylyl cyclase activity. Activation of the $\mathrm{G}_{\alpha \mathrm{i} / \mathrm{o}}$ results in $\mathrm{G}_{\text {ai/o }}$-mediated inhibition of adenylyl cyclase causing a reduced production of cyclic adenosine monophosphate (cAMP).

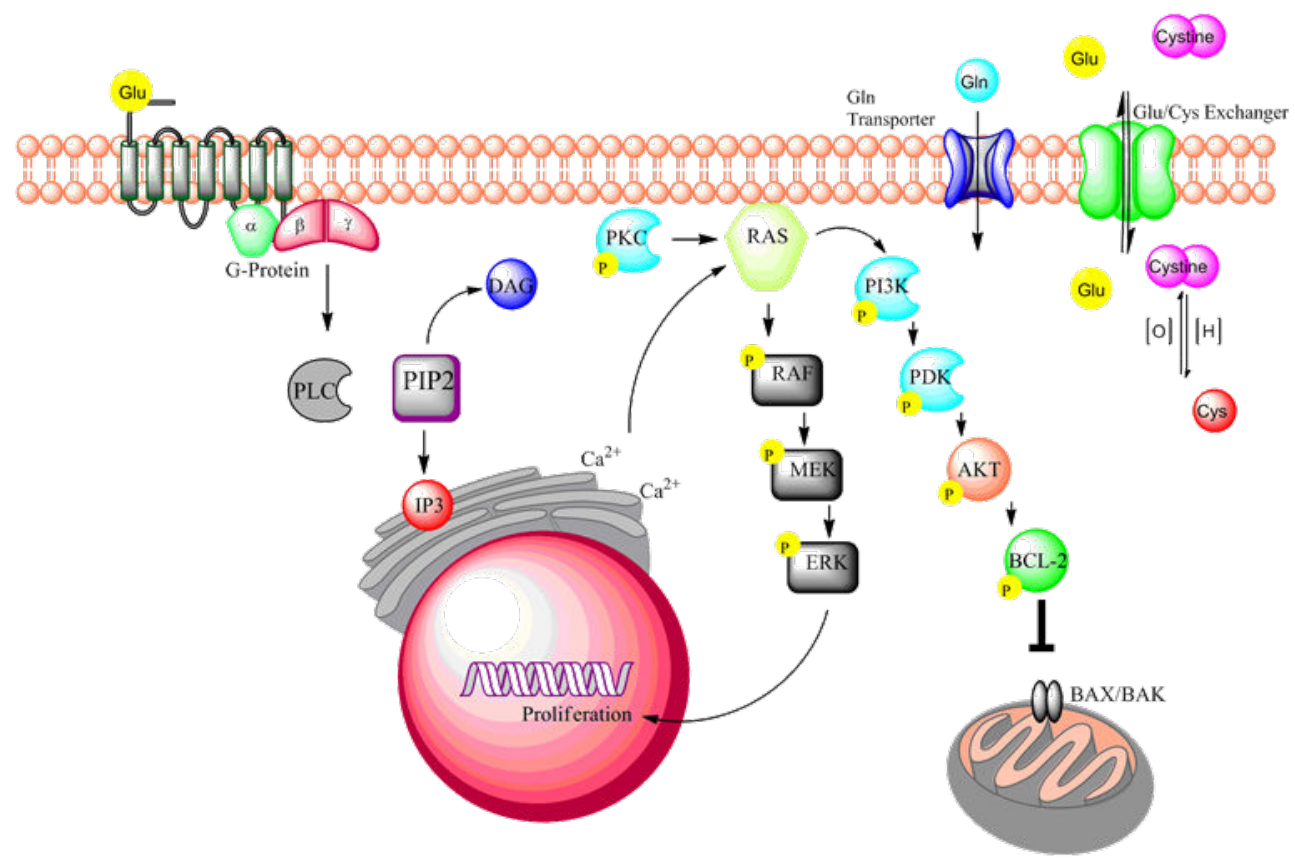

Figure 2. Signal transduction pathways activated by mGluR1 stimulation. Activation of mGluR1 by its ligand, glutamate, results in the activation of signaling pathways that upregulate cell proliferation (MAPK) and promotes cell survival (PI3K/AKT).

\subsection{G-protein coupled receptors and cellular transformation}

GPCRs comprise the largest known family of cell surface receptors that mediate cellular responses to a diverse array of signaling molecules including hormones, neurotransmitters, and chemokines. In addition, potent mitogens such as thrombin, bombesin, lysophosphatidic acid (LPA), gastrin-releasing peptide (GRP), endothelin and prostaglandins can stimulate cell proliferation by interacting with their cognate GPCR in various homologous cell types [18, 19, 20, 21, 22].

A fundamental trait of cancer cells involves their ability to sustain chronic proliferation. In normal cells, the production and release of growth-promoting signals that allow entry and progression through the cell cycle are tightly regulated. This regulation maintains cellular 
homeostasis promoting normal tissue structure and function and is controlled, in part, by growth factors that bind cell-surface receptors, which usually transmit signals through branched signaling pathways. By deregulating these signals, cancer cells are able to bypass regulatory control allowing them to proliferate and grow unconditionally. Cancer cells can acquire the ability to sustain proliferative signaling by producing these growth factors themselves in which the cells respond through the expression of cognate receptors resulting in autocrine proliferative stimulation. Alternatively, cancer cells may send signals to the surrounding tissue in a paracrine-like manner causing them to release various growth factors that promote growth of the cancer cells [23]. Activation of the surface receptors may also cause the constitutive activation of downstream signaling pathways.

Overexpression of GPCRs is detected in various cancer types, contributing to tumor cell growth once activated by circulating or locally produced ligands. The oncogenic potential of $\mathrm{G}$ protein-coupled receptors was revealed with the discovery of the MAS receptor in 1986, which provided a direct link between misregulated cell growth and GPCRs [24]. In contrast to most oncogenes identified at that time, MAS did not harbor genetic mutations resulting in its constitutive activation. Subsequent to its discovery, studies demonstrated that wild-type GPCRs have the ability to become tumorigenic when exposed to an excess of locally produced or circulating ligands and agonists while other GPCRs harboring mutations in key conserved residues can have transforming activity even in the absence of their ligands [14, 16, 17, 25, 26, $27,28]$. It has also been found that the level of expression of GPCRs is not as imperative to oncogenesis as is the receptor simply being expressed [28]. Since then, abnormal functioning of GPCR pathways have been shown to be the cause of several human diseases, and a large body of evidence links aberrant G-protein signaling to the development and progression of certain cancers [17].

The oncogenic transformation of cell types by way of GPCRs occurs, in part, by the establishment of autocrine or paracrine feedback in which the cell releases the ligand which binds and activates the receptor, maintaining its activation, as well as that of downstream effector proteins leading to enhanced cell proliferation $[29,30]$. mGluRs are members of GPCR with glutamate as the natural ligand [31,32]. Glutamate is the predominant excitatory neurotransmitter in the central nervous system and stimulates both ionotropic (ligand-gated ion channels) and metabotropic (G-protein-coupled) glutamate receptors [33]. Previously, glutamate signaling was believed to be exclusive to the central nervous system (CNS). It has since become apparent that various peripheral, non-neuronal cells express different types of glutamate receptors and these receptors are required for normal physiological function [34]. Studies have demonstrated that not only are mGluRs involved in the normal maintenance and regulation of physiological homeostasis, but they can also be involved in the progression of a variety of human malignancies. Initially, because of prior implications of GPCR expression being exclusive to the CNS, the first speculations of mGluRs in human cancers were in neuronal tumors such as neuroblastoma, medulloblastoma and glioma [35, 36, 37]. It was found that among these neuronal tumors that released excess amount of glutamate into the surrounding microenvironment were growing at a much-increased rate compared to neuronal tumors that were not secreting glutamate into their surroundings [38]. Subsequent studies revealed that 
inhibition of glutamate release correlated with a decrease in the proliferation of not only neuroblastoma, rhabdomyosarcoma, brain astrocytoma, but also thyroid carcinoma, lung carcinoma, colon adenocarcinoma and breast carcinoma while having no effect on the growth rates of normal human fibroblast or bone marrow stromal cells [37].

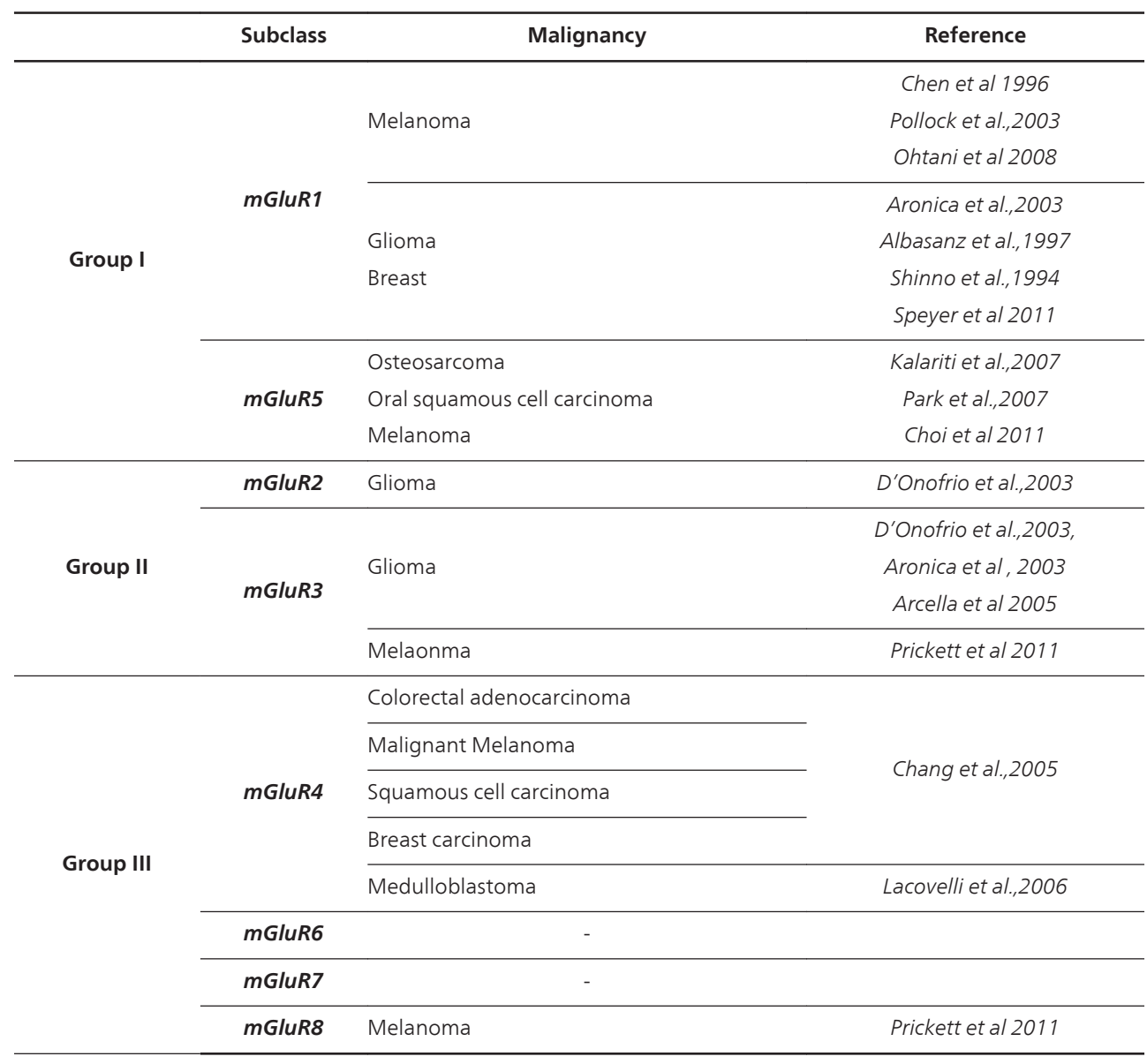

Table 2. Implications of mGluRs in human cancer

\subsection{Ectopic expression of mGluR1}

Our group has previously described an unknown mechanism of melanoma pathogenesis in which the expression of an otherwise normal receptor, metabotropic glutamate receptor 1 (mGluR1) in an unnatural cellular environment (melanocytes) is able to upregulate cell proliferation and tumor development in vitro and in vivo. Chen and colleagues have established a transgenic mouse line (TG-3) that spontaneously develops melanoma with $100 \%$ 
penetrance that was constructed using a 2-kb genomic fragment, termed clone $\mathrm{B}$, which was previously shown to commit fibroblasts to undergo adipocyte differentiation $[39,40]$. In an attempt to establish an animal model of obesity, five founder animals were established using clone B DNA: TG-1 through -5. Instead of the expected obese phenotype, one of the five founders, TG-3, developed heavily pigmented lesions on the ears, around the eyes, and the perianal region at approximately eight months of age. Upon histological analyses these pigmented lesions were identified as melanoma, Further examination shown these lesions were metastatic as evident by detecting these pigmented lesions in distant organs including the lymph nodes, brain, bone, lung and muscle. Using physical mapping we identified a single insertion of clone $\mathrm{B}$ in mouse chromosome 10, region A2, which is orthologous to human chromosome 6q23-24. Further molecular mapping found a concurrent insertion of clone $\mathrm{B}$ in a head-to-head, head-to-tail, tail-to-tail configuration and a deletion of approximately $70-\mathrm{kb}$ of the host sequences located in intron 3 of the gene encoding mGluR1. Assessment of mGluR1 at both the protein and mRNA levels revealed the expression of the murine form of metabotropic glutamate receptor 1 only in the pigmented lesion but not normal counterpart. These results suggested that ectopic expression of mGluR1 in melanocytes might be the driving basis for melanoma development [41]. To verify this hypothesis, we engineered a new transgenic mouse line [TG(Grm1)EPv] directing mGluR1 expression in melanocytes under the control of a melanocyte-specific promoter, dopachrome tautomerase (DCT). This new transgenic line displays a melanoma susceptibility phenotype and progression very similar to the TG-3 line [42]. It was concluded that in vivo, aberrant mGluR1 expression in melanocytes is sufficient to induce spontaneous melanoma development with $100 \%$ penetrance. Progeny that are heterozygous at the transgenic integration site develop melanotic lesions as soon as 4 months post-natal while those homozygous at the integration site develop lesions within 30 days after birth. Ohtani and co-workers confirmed our observation with an inducible mGluR1 transgenic mouse model, when the expression of mGluR1 is conditionally activated in adult mice melanoma develops in these animals $100 \%$ of the time [43]. The results that mGluR1 has a role in melanocytic neoplasia in the murine system prompted our group to examine human melanoma for ectopic expression of the human form of metabotropic glutamate receptor 1. Examination of numerous melanoma biopsies and cell lines demonstrated aberrant expression of the receptor in approximately $60 \%$ of the samples suggesting that mGluR1 may be involved in the oncogenesis of a subset of human melanomas $[41,44]$. These findings as well as those by others prompted our group to explore the underlying mechanisms of mGluR1- mediated melanocytic transformation. In a set of studies we confirmed that mGluR1 expression in melanocytes confers oncogenic activities in vitro and is sufficient to cause malignant phenotype in vivo [45].

In both the murine and human melanoma cell lines, stimulation of mGluR1 by glutamate results in similar if not identical formation of second messengers as described above for the CNS. One of the second messengers, DAG, stimulates PKC. PKC plays a key role in a multitude of cellular processes including apoptosis, malignant transformation, and metastasis [46] and once it is activated it in turn can activate the MAPK and PI3K/AKT pathways [47, 48, 49, 50, 51]. It is well known that the RAS-RAF-MEK-ERK module of the MAPK signaling cascade regulates cell proliferation and inhibits apoptosis. PI3K/AKT pathway activation is important 
for tumor cell survival, epithelial-mesenchymal transition (EMT), and angiogenesis [52]. In addition, gain-of-function mutations causing constitutive activation in G-proteins also result in the activation of downstream effector pathways leading to uncontrolled cell proliferation and cell survival. This is often a consequence of specific amino acid substitutions in the GTP binding region of the G $\alpha$ subunit resulting in defective GTPase activity. The inability for GTP to be cleaved to GDP allows constitutive activation of downstream effector molecules. Such hypermorphic mutant $G$ proteins in melanocytes were initially found to cause increased dermal pigmentation in transgenic mice. It was later found that the two functionally related $\mathrm{G}$ protein subunits, GNAQ and GNA11 encoding the stimulatory $G \alpha_{\mathrm{q}}$ and $G \alpha_{11}$ subunits of heterotrimeric G-proteins respectively, are genetic hotspots for somatic mutations found in early uveal melanoma pathogenesis [53]. These mutations were also detected in benign intradermal melanocytic tumors as well as primary and metastatic ocular melanocytic tumors. Therefore, activation of ectopically expressed metabotropic glutamate receptors in human cells upregulate signaling pathways known to be important in the pathogenesis of human cancers associated with increased growth and angiogenesis while interruption of such pathways would result in a decrease in cell proliferation and survival.

The acquisition of tumor phenotypes is dependent not on one, but on successive alterations in the genomes of neoplastic cells. Specific genotypes confer selective advantages on subclones of cells enabling them to outgrow, and eventually dominate, the local tissue environment. This idea of multistage tumor progression can be viewed as a succession of clonal expansion each triggered by chance acquisition of mutant genotypes transitioning a cell from normal through pre-malignant and eventually cancer. One stage involved in malignant transformation of cell types is the ability of a cell to maintain its capacity to spread and divide in the absence of a stable or inter surface to attach. In a study performed by Prickett et al, exon capture sequencing of GPCRs in malignant melanoma specimens identified activating mutations within a region encoding for a member of the group II metabotropic glutamate receptors, mGluR3. The mutations were found throughout the coding region and affected the extracellular domains as well as the seven-transmembrane domain with two minihotspots located proximal to the transmembrane domains. Four somatic mutations (mGluR3 ${ }^{\mathrm{E} 767 \mathrm{~K}}$, mGluR3 ${ }^{\mathrm{S} 610 \mathrm{~L}}, \mathrm{mGluR} 3^{\mathrm{G} 561 \mathrm{E}}$, and $\mathrm{mGluR}^{\mathrm{E} 870 \mathrm{~K}}$ ) were found to selectively regulate the phosphorylation of MEK1/2 kinase in vivo and induce micro metastasis in vivo. This activation resulted in the increased migration and anchorage independence growth of melanoma cells. These studies suggest the existence of a melanoma tumor type in which activating mutations in mGluR3 can lead to hypersensitivity to agonist stimulation of the MEK-MAPK pathway that bypasses the RAF/RAS genotypes.

In addition to melanoma, inappropriate glutamatergic signaling has been shown to be sufficient in the dysregulating growth leading to the transformation of other cell types. The ectopic expression of mGluR1 alone was able to induce the neoplastic transformation of immortalized baby mouse epithelial cells (iBMK) in vitro and promote tumor cell proliferation in vivo $[54,55]$. The iBMK cell model was engineered to be immortal but not tumorigenic while also retaining normal epithelial characteristics. It was shown that full-length wild-type mGluR1 is tumorigenic when ectopically expressed in epithelial cells via stimulated MAPK 
and AKT signaling pathways. Additionally, studies performed by Speyer and colleagues (2011) described mGluR1 expression in several triple-negative breast cancer cell lines (TNBC) in which shRNA-mediated knockdown of mGluR1 expression as well as antagonists to the receptor inhibited cell proliferation in TNBC lines. Malignancies involving other mGluRs include mGluR4 first reported by Chang and colleagues that mGluR4 is overexpressed in more than $40 \%$ of colorectal adenocarcinomas, malignant melanomas, laryngeal squamous cell carcinomas, and breast carcinomas tested [56]. Among these cancers, there was a correlation with mGluR4 overexpression and increased mortality rate in patients with colorectal carcinoma. mGluR5 overexpression was found to induce melanoma in transgenic mice [57]. The increased expression in mGluR5 in the mouse melanocytes was correlated with enhanced levels of MAPK activation. These studies suggested that glutamatergic signaling plays a significant role in the initiation, progression and maintenance in human malignancies.

\section{4. mGluR1 expression is required to maintain transformed phenotype}

In an attempt to maintain tumor phenotypes, cancer cells are able to limit or bypass apoptotic cell response. In addition to manipulating cellular signals that control proliferation, oncogenic mutations have a profound effect in the disruption of apoptosis. These disruptions cause evasion of death response signals and have been shown to promote tumor initiation and progression leading to multistage carcinogenesis [58, 59, 60]. Indeed, defects in apoptotic pathways contribute to a number of human disease phenotypes from neurodegeneration to malignancy. The phosphatidylinositol 3-kinase (PI3K), phosphatase and tensin homolog deleted on chromosome 10 (PTEN) have critical roles in the transmission of signals that control multiple functions such as cell metabolism, proliferation, cell-cycle progression, and survival. PI3Ks are divided into three types (type I, II and III) with type I being further divided into two sub classes, class IA and IB. PI3Ks belonging to class IA are activated by receptor tyrosine kinases (RTKs) and those classified in class IB are activated by GPCRs [61]. All type I PI3Ks catalyze the conversion of the lipid phosphatidylinositol-4,5-bisphosphate (PIP2) to phosphatidylinositol-3,4,5-triphosphate (PIP3) from activation of upstream receptors such as epidermal growth factor receptor (EGFR), fibroblast growth factor receptor (FGFR), platelet-derived growth factor receptor (PDGFR) and insulin-like growth factor 1 receptor (IGF-1R), which interact with the p85 regulatory subunit of PI3K $[62,63]$. The Ras protein interacts by directly activating p110 catalytic subunit of PI3K in a GTP-dependent manner. The activation of Ras is regulated by the extracellular stimuli that interact with cell surface receptors of the tyrosine kinase or seven transmembrane-spanning families [64]. Once activated Ras phosphorylates MEK, the mitogen activated protein (MAP) kinase kinase, which in turn activates MAP kinase. This cascade stimulates the MAP kinase pathway leading to phosphorylation of transcription factor that promote cellular proliferation and survival.

\subsection{PI3K/AKT/PTEN}

The serine-threonine protein kinase, AKT (also known as protein kinase B or PKB) and phosphoinositide-dependent kinase 1 (PDK1) are important downstream targets of PI3K. The 
main biological consequences of AKT activation are cell survival and increased cell growth. AKT activation is known to involve in both mitogenic signaling and cell survival in a number of human cancer types. Human AKT exists in three isoforms: AKT1, AKT2 and AKT3. PI3K mediates cleavage of $\mathrm{PIP}_{2}$, which produce $\mathrm{PIP}_{3}$ at the inner surface of the plasma membrane. PIP which acts as a second messenger to recruit AKT through interaction of its pleckstrin homology $(\mathrm{PH})$ domain, a domain conserved in signaling proteins that accumulate at sites of PI3K activation. Phosphorylation of AKT is dependent on phosphorylation, in part, by PDK1 [61, 65]. Activated AKT in turn mediates signaling through the phosphorylating inactivation of a multitude of downstream targets involved in apoptosis, including the phosphorylation of cyclic AMP response element--binding protein (CREB) as well as I $\kappa B$ kinase (IKK), which further activates central regulators of cell death: nuclear factor $-\kappa B$ (NF$\kappa \mathrm{B})$ and p53. AKT-mediated induction of NF- $\kappa \mathrm{B}$ occurs via activation of I $\kappa$ B which induces degradation of the NF- $\kappa \mathrm{B}$ inhibitor I $\kappa \mathrm{B}$ leading to release of NF- $\kappa \mathrm{B}$ from the cytoplasm and translocation to the nucleus leading to the expression of survival genes such as IAP that promotes survival by inhibiting downstream activation of caspase-9 and -3 [66, 67, 68, 69, 70]. AKT influences p53 activity through phosphorylation of the p53-binding protein, MDM2. MDM2 regulates p53 function by targeting p53 for degradation by its E3 ubiquitin ligase. AKT phosphorylation of MDM2 allows increased translocation of the proteasome to the nucleus where it binds p53 resulting in its degradation [70, 71]. In addition, the oncological significance of amplified AKT expression has been well documented in gastric carcinoma, ovarian, pancreatic and breast tumors $[72,73,74]$. AKT phosphorylation of the apoptosisinducing protein, $\mathrm{Bad}$, prevents binding of $\mathrm{Bad}$ to the antiapoptotic $\mathrm{Bcl}-2$-type proteins, $\mathrm{Bcl}-2$ and Bcl- $x_{L}$. Bad promotes cell death by forming a non-functional hetero-dimer with the survival factor $\mathrm{Bcl}-\mathrm{x}_{\mathrm{L}}$. Upregulation of $\mathrm{Bcl}-\mathrm{x}_{\mathrm{L}}$ promotes survival by regulating coupled respiration through supporting ATP/ADP exchange across the outer mitochondrial membrane [75]. Phosphorylation of Bad by AKT prevents heterodimerization with Bcl- $\mathrm{x}_{\mathrm{L}}$ restoring its antiapoptotic function. In addition, it has also been implicated that PI3K-AKT has effects on tumor-induced angiogenesis, mediated in part through hypoxia-induced factor- $1 \alpha$ and $\mathrm{NF}-\kappa \mathrm{B}$ induced expression of the proangiogenic growth factor, vascular endothelial growth factor (VEGF) and suppression the endogenous antiangiogenic molecule, thrombospondin-1 (TSP-1). VEGF and its receptor, VEGFR are major angiogenesis inducers associated with tumor angiogenesis in numerous malignancies [76].

The PTEN tumor suppressor is a dual-specificity phosphatase, which has protein phosphatase activity and lipid phosphatase activity that antagonizes the PI3K-AKT pathway and is found to contain inactivating mutations in many human cancers. PTEN negatively regulates $\mathrm{PIP}_{3}-$ mediated signaling cascades by hydrolyzing the 3-phosphate to generate $\mathrm{PIP}_{2}$ making PTEN critical in phosphatidylinositol homeostasis and regulating the proliferation and invasion of many cancer cells [77].

AKT is an important regulator of cell growth and apoptosis that activate downstream from PI3K and is inhibited by the actions of the tumor suppressor PTEN. The ability to promote cell growth and suppress cell death suggests the PI3K/AKT /PTEN pathways are critical participants in tumorigenesis. As previously stated, the ectopic expression of mGluR1 was detected 
in approximately $60 \%$ of human melanoma biopsies and cell lines examined while its expression was not found in in normal skin or benign nevi $[42,44]$. Stimulation of mGluR1 by an agonist of the receptor, L-quisqualate, results in the activation of MAPK in mouse and human melanoma cell lines independent of the presence of the most common NRAS/BRAF-activating mutations [46]. Constitutively activated MAPK pathway is common in many human cancers including melanoma, frequently due to mutations in the BRAF and RAS genes. In melanomas, mutation rates for BRAF is $\sim 60 \%$ and NRAS is $\sim 20 \%[44,78,79]$. Treatment of human melanoma cells with a competitive antagonist of mGluR1, LY367385, which binds to the same catalytic site as glutamate, showed dose-dependent inhibition of cell proliferation via suppression of ERK activation [80]. In addition, a noncompetitive mGluR1 antagonist which binds to the transmembrane domain of the receptor resulting in stabilization of inactive conformation, BAY36-7620, suppressed cell growth and promoted apoptosis in cultured melanoma cells that express mGluR1 [81]. Both LY367385 and BAY36-7620 were shown to result in a decrease in the levels of the secondary messengers, $\mathrm{IP}_{3}$ and DAG, in these studies. Further investigation of mGluR1 functionality and activity using dominant-negative mGluR1 (dn-mGluR1) mutant yielded in an increase in the number of apoptotic cells as demonstrated by the increased cleavage of poly-ADP ribose polymerase (PARP), a well-known marker of apoptosis. The dnmGluR1 mutants derived from small deletions or single base substitutions in the intracellular loop 2 or 3, which have been shown previously to be essential in mGluR1 signaling [44, 46, 82]. These studies demonstrated that ablation of the functionality of mGluR1 in melanoma cells, we were able to reduce cell proliferation and induce cell death in vitro without affecting normal cell types [44].

\section{Inhibition of mGluR1}

Targeting strategies for cancer therapy differ from chemotherapy and radiotherapy in their potential to provide tumor specificity. Preclinical studies have demonstrated that inhibition of glutamatergic signaling results in decreased tumor cell growth and increased cell death. Identifying key upstream molecular targets that lead to the activation of proliferation and survival pathways is important in cancer drug discovery. Gene-targeting techniques that selectively inactivate gene expression assist in the validation of novel proteins involved in disease pathogenesis.

\subsection{Suppression of mGluR1 by siRNA}

Gene silencing by short-interfering RNAs (siRNAs) has been used to investigate their roles in different cell types and in various organisms. However, constitutive suppression the expression of a gene could have deleterious consequences therefore, inducible silencing RNA approaches could be useful by relying on small molecules that act as inducers to modify synthetic transcription factors thereby regulating the expression of a target gene [83-87]. The tetracycline operon based tetracycline inducible system and the non-mammalian steroid based ecdysone inducible system are two complementary approaches we have used. Previous attempts to inhibit mGluR1 expression in human melanoma cells using constitutively ex- 
pressed mGluR1 specific siRNAs resulted in the cells exhibiting a dormancy-like state prior to dying. Employing the inducible knock-down of mGluR1 was shown to result in suppression of MAPK as evident by a decrease in the phosphorylated form of ERK as well as inhibition of the PI3/AKT cell survival pathway seen by the reduced form of activated AKT [84]. Our previous studies on mouse melanoma cells as well as mouse melanocytic clones that stably expressed mGluR1 showed elevated levels of glutamate were released into their surroundings compared to normal mouse melanocytes or vector controls [85]. This finding correlated with earlier works in rats in which suppression of glutamate uptake coupled with stimulation of group I mGluRs results in an increase in the accumulation of extracellular glutamate. It was implicated that these mGluRs may work systematically with cystine-glutamate exchangers to regulate extracellular glutamate concentrations [86, 87]. From this discovery, Chen and coworkers hypothesized that instead of inhibiting mGluR1 directly, if they were able to interrupt the autocrine/paracrine activity by limiting the amount of free ligand this would result in a similar decrease in cell proliferation/survival.

\subsection{Suppression of mGluR1 using an inhibitor of glutamate release}

Glutamate is a major excitatory neurotransmitter in the CNS. The over activation of neurons by excessive glutamate stimulation is excitotoxic, resulting in neuronal death [88]. Interestingly, the brain is one of the most common sites of metastatic melanoma. It is possible the excessive glutamate found in the brain promotes tumor growth while inducing excitotoxicity to the surrounding neuronal tissue. Because of this, Chen and co-workers took advantage of using an inhibitor of glutamate release, riluzole, a Food and Drug Administration (FDA)-approved drug for the treatment of amyotrophic lateral sclerosis (ALS or Lou Gehrig's disease). ALS is a degenerative motor neuron disease of the upper and lower motor neurons that progressively causes weakness, muscle atrophy and fasciculation. Riluzole is known to inhibit the release of glutamate from the presynapse resulting in a decrease of the molecule in the synaptic cleft resulting in a decrease in excitotoxicity caused by the neurotransmitter. This leads to a decreased progression of ALS [89]. Riluzole treated melanoma cells showed a reduction in the levels of glutamate in the surroundings in vitro, which correlated with a decrease in cell growth in vivo when compared to normal cells exposed to the drug. Additionally, melanoma cells treated with relatively low concentrations of riluzole were shown to undergo apoptosis by an increase in the levels of the cleaved form of PARP after riluzole treatment. The therapeutic potential of riluzole was validated in vivo using a human melanoma cell xenograft model where animals were treated with a human equivalent of the maximally allowed daily amount of riluzole per body weight per day via p.o. gavage for 18 days. The use of riluzole as a putative antagonist of mGluR1 to inhibit glutamate signaling resulted in a reduction in tumor volume in treated animals vs. control. These results were translated to the clinic.

\subsection{Translational study in patients}

Clinical trials involving new compounds are classified into four phases: Phase 0, I, II and III. Phase 0 is the designation for exploratory, first-in-human trials conducted with the U.S. 
FDA's guidelines on Exploratory Investigational New Drug Studies (IND). Phase 0 trials are designed to speed-up the development of promising drugs or imaging agents by establishing very early on whether the drug or agent behaves in human subjects as was anticipated from preclinical studies. Based on results from previous findings in preclinical studies using riluzole, a phase 0 trial using the compound in patients with stage III and IV melanoma was performed to see if in itds presence the target, glutamatergic signaling, is reduced. The trial resulted in a significant short-term response rate of $34 \%$ of patients. Tumors from patients that displayed shrinkage of tumors showed an inhibition of signaling through both the MAPK and PI3K/AKT pathways, reflecting results that were previously observed in preclinical studies [44, 90]. Positron Emission tomography (PET) documented complete resolve of multiple nodal and cutaneous metastases in several patients. One patient having six different tumors measured by PET/CT demonstrated shrinkage of the lesions by $10 \%$ to $20 \%$. Out of eleven patients that completed the study, only two had shown progression of the disease after treatment [90]. The tumor samples were a composed of patients harboring both V600E BRAF as well as Q61K NRAS mutations. In addition all the patients admitted into the trial expressed mGluR1, although mGluR1 expression status was not a requirement for entry. It is possible that, from this observation, that more advanced tumors, those found in stage III and IV metastases, have a higher frequency of mGluR1 expression and that ectopic mGluR1 occurs and is more frequent in more progressed stages of the disease.

\section{Conclusion}

Melanoma is a genetically diverse disease owing to the dysfunction of multiple regulatory pathways. As such, it is important to test the therapeutic potential of agents that reflect the genetic diversity of this disease. In addition to ectopic expression of mGluR1, mutations in BRAF have been identified in more than $60 \%$ of melanomas, most of these due to the substitution of a single amino acid at residue 600 in the B-RAF kinase domain resulting in constitutive activation of the RAF/MAP/ERK kinase signaling pathway [91]. Melanoma cells with aberrant glutamate receptor signaling also exhibit hyperactive PI3K/AKT and MEK/MAPK pathways [44, 92, 93]. Additionally, a recent examination of a publicly available gene expression data bank identified a variety of GPCRs that are over-expressed in diverse types of cancer tissues [94]. These findings illustrate the role that glutamate receptors and transporters play in the cellular signaling somatic cell types. The molecular physiology of GPCRs allow them to be regulated by many agonists and antagonists and are the target, directly and indirectly, of approximately $50 \%$ of pharmaceutical agents used to treat various human diseases. GPCRs have been shown to have a role in both normal and aberrant mitogenic signaling in functions that regulate cell migration in tumor metastasis and angiogenesis as well as key molecular events implicated in cancer progression and invasion. Based on these findings, it is perhaps not surprising that interruption of the glutamatergic signaling pathway by targeting GPCRs in cancers could result in more effective the treatment of this devastating disease. 


\section{Author details}

Brian A. Wall ${ }^{1,2}$, Seung-Shick Shin ${ }^{3}$ and Suzie Chen ${ }^{1,2,3}$

1 Susan Lehman Cullman Laboratory for Cancer Research, Ernest Mario School of Pharmacy, Rutgers University, NJ, USA

2 Joint Graduate Program of Toxicology, Rutgers, the State University, Piscataway, NJ, USA

3 The Cancer Institute of New Jersey, Little Albany Street, New Brunswick, NJ, USA

\section{References}

[1] Warburg, O. On the origin of cancer cells. Science (1956). Feb 24;, 123(3191), 309-14.

[2] Warburg, O. On respiratory impairment in cancer cells. Science (1956). Aug 10; 124(3215), 269-70.

[3] Warburg, O, Wind, F, \& Negelein, E. The Metabolism of Tumors in the Body. J Gen Physiol (1927). Mar 7; 8(6), 519-30.

[4] Moreadith, R. W, \& Lehninger, A. L. The pathways of glutamate and glutamine oxidation by tumor cell mitochondria. Role of mitochondrial NAD $(\mathrm{P})+$ +-dependent malic enzyme. J Biol Chem (1984). May 25;, 259(10), 6215-21.

[5] Deberardinis, R. J, Sayed, N, Ditsworth, D, \& Thompson, C. B. Brick by brick: metabolism and tumor cell growth. Curr Opin Genet Dev (2008). Feb;, 18(1), 54-61.

[6] Deberardinis, R. J, Mancuso, A, Daikhin, E, Nissim, I, Yudkoff, M, Wehrli, S, et al. Beyond aerobic glycolysis: transformed cells can engage in glutamine metabolism that exceeds the requirement for protein and nucleotide synthesis. Proc Natl Acad Sci U S A (2007). Dec 4; 104(49), 19345-50.

[7] Estrela, J. M, Ortega, A, \& Obrador, E. Glutathione in cancer biology and therapy. Crit Rev Clin Lab Sci (2006). , 43(2), 143-81.

[8] Feron, O. Pyruvate into lactate and back: from the Warburg effect to symbiotic energy fuel exchange in cancer cells. Radiotherapy and oncology : journal of the European Society for Therapeutic Radiology and Oncology (2009). Sep;, 92(3), 329-33.

[9] Semenza, G. L. Tumor metabolism: cancer cells give and take lactate. J Clin Invest (2008). Dec;, 118(12), 3835-7.

[10] Fredriksson, R, Lagerstrom, M. C, Lundin, L. G, \& Schioth, H. B. The G-protein-coupled receptors in the human genome form five main families. Phylogenetic analysis, paralogon groups, and fingerprints. Mol Pharmacol (2003). Jun;, 63(6), 1256-72. 
[11] Bjarnadottir, T. K, Fredriksson, R, \& Schioth, H. B. The gene repertoire and the common evolutionary history of glutamate, pheromone (taste(1) and other related G protein-coupled receptors. Gene (2005). Dec 5;362:70-84., 2R

[12] Hara, O, Sheppard, P. J, Thogersen, P. O, Venezia, H, Haldeman, D, \& Mcgrane, B. A. $\mathrm{V}$, et al. The ligand-binding domain in metabotropic glutamate receptors is related to bacterial periplasmic binding proteins. Neuron (1993). Jul;, 11(1), 41-52.

[13] Kaupmann, K, Huggel, K, Heid, J, Flor, P. J, Bischoff, S, Mickel, S. J, et al. Expression cloning of GABA(B) receptors uncovers similarity to metabotropic glutamate receptors. Nature (1997). Mar 20;, 386(6622), 239-46.

[14] Hamm, H. E. The many faces of G protein signaling. J Biol Chem (1998). Jan 9;, 273(2), $669-72$.

[15] Bunemann, M, Frank, M, \& Lohse, M. J. Gi protein activation in intact cells involves subunit rearrangement rather than dissociation. Proc Natl Acad Sci U S A (2003). Dec $23 ; 100(26), 16077-82$.

[16] Lappano, R, \& Maggiolini, M. G protein-coupled receptors: novel targets for drug discovery in cancer. Nat Rev Drug Discov (2011). Jan; 10(1), 47-60.

[17] Lee, H. J, \& Wall, B. Chen S. G-protein-coupled receptors and melanoma. Pigment Cell Melanoma Res (2008). Aug;, 21(4), 415-28.

[18] Rozengurt, E, Guha, S, \& Sinnett-smith, J. Gastrointestinal peptide signalling in health and disease. Eur J Surg Suppl (2002). , 587, 23-38.

[19] Teerawattananon, Y, Tangcharoensathien, V, Tantivess, S, \& Mills, A. Health sector regulation in Thailand: recent progress and the future agenda. Health Policy (2003). Mar; 63(3), 323-38.

[20] Marinissen, M. J. Gutkind JS. G-protein-coupled receptors and signaling networks: emerging paradigms. Trends Pharmacol Sci (2001). , 22(7), 368-76.

[21] Dorsam, R. T. Gutkind JS. G-protein-coupled receptors and cancer. Nat Rev Cancer (2007). Feb; 7(2), 79-94.

[22] Rzeski, W, Turski, L, \& Ikonomidou, C. Glutamate antagonists limit tumor growth. Proc Natl Acad Sci U S A (2001). May 22;, 98(11), 6372-7.

[23] Cheng, N, Chytil, A, Shyr, Y, Joly, A, \& Moses, H. L. Transforming growth factorbeta signaling-deficient fibroblasts enhance hepatocyte growth factor signaling in mammary carcinoma cells to promote scattering and invasion. Mol Cancer Res (2008). Oct; $6(10), 1521-33$.

[24] Young, D, Waitches, G, Birchmeier, C, Fasano, O, \& Wigler, M. Isolation and characterization of a new cellular oncogene encoding a protein with multiple potential transmembrane domains. Cell (1986). Jun 6; 45(5), 711-9. 
[25] Li, F, Wang, Y, Zeller, K. I, Potter, J. J, Wonsey, D. R, \& Donnell, O. KA, et al. Myc stimulates nuclearly encoded mitochondrial genes and mitochondrial biogenesis. Mol Cell Biol (2005). Jul;, 25(14), 6225-34.

[26] Lundstrom, K. Latest development in drug discovery on G protein-coupled receptors. Curr Protein Pept Sci (2006). Oct;, 7(5), 465-70.

[27] Knox, W. E, Horowitz, M. L, \& Friedell, G. H. The proportionality of glutaminase content to growth rate and morphology of rat neoplasms. Cancer Res (1969). Mar; 29(3), 669-80.

[28] Van Raamsdonk, C. D, Bezrookove, V, Green, G, Bauer, J, Gaugler, L, \& Brien, O. JM, et al. Frequent somatic mutations of GNAQ in uveal melanoma and blue naevi. $\mathrm{Na}$ ture (2009). Jan 29;, 457(7229), 599-602.

[29] Burger, M, Burger, J. A, Hoch, R. C, Oades, Z, Takamori, H, \& Schraufstatter, I. U. Point mutation causing constitutive signaling of CXCR2 leads to transforming activity similar to Kaposi's sarcoma herpesvirus-G protein-coupled receptor. J Immunol (1999). Aug 15;, 163(4), 2017-22.

[30] Takayama, H. LaRochelle WJ, Sharp R, Otsuka T, Kriebel P, Anver M, et al. Diverse tumorigenesis associated with aberrant development in mice overexpressing hepatocyte growth factor/scatter factor. Proc Natl Acad Sci (1997). , 94, 701-6.

[31] Pin, J. P, \& Duvoisin, R. The metabotropic glutamate receptors: structure and functions. Neuropharmacology (1995). Jan;, 34(1), 1-26.

[32] Hermans, E, \& Challiss, R. A. Structural, signalling and regulatory properties of the group I metabotropic glutamate receptors: prototypic family C G-protein-coupled receptors. Biochem J (2001). Pt 3):465-84.

[33] Ozawa, S, Kamiya, H, \& Tsuzuki, K. Glutamate receptors in the mammalian central nervous system. Prog Neurobiol (1998). Apr;, 54(5), 581-618.

[34] Haas, H. S, Pfragner, R, Siegl, V, Ingolic, E, Heintz, E, Schraml, E, et al. The non-competitive metabotropic glutamate receptor-1 antagonist CPCCOEt inhibits the in vitro growth of human melanoma. Oncol Rep (2007). Jun; 17(6), 1399-404.

[35] Naarala, J, Nykvist, P, Tuomala, M, \& Savolainen, K. Excitatory amino acid-induced slow biphasic responses of free intracellular calcium in human neuroblastoma cells. FEBS Lett (1993). Sep 13; 330(2), 222-6.

[36] Iacovelli, L, Fulceri, F, De Blasi, A, Nicoletti, F, Ruggieri, S, \& Fornai, F. The neurotoxicity of amphetamines: bridging drugs of abuse and neurodegenerative disorders. Exp Neurol (2006). Sep; 201(1), 24-31.

[37] Shin, S. S, Martino, J. J, \& Chen, S. Metabotropic glutamate receptors (mGlus) and cellular transformation. Neuropharmacology (2008). Sep; 55(4), 396-402. 
[38] Takano, T, Lin, J. H, Arcuino, G, Gao, Q, Yang, J, \& Nedergaard, M. Glutamate release promotes growth of malignant gliomas. Nat Med (2001). Sep; 7(9), 1010-5.

[39] Chen, S, Zhu, H, Wetzel, W. J, \& Philbert, M. A. Spontaneous melanocytosis in transgenic mice. J Invest Dermatol (1996). May;, 106(5), 1145-51.

[40] Zhu, H, Reuhl, K, Zhang, X, Botha, R, Ryan, K, Wei, J, et al. Development of heritable melanoma in transgenic mice. J Invest Dermatol (1998). Mar;, 110(3), 247-52.

[41] Pollock, P. M, Cohen-solal, K, Sood, R, Namkoong, J, Martino, J. J, Koganti, A, et al. Melanoma mouse model implicates metabotropic glutamate signaling in melanocytic neoplasia. Nat Genet (2003). May; 34(1), 108-12.

[42] Pollock, P. M, Cohen-solal, K, Sood, R, Namkoong, J, Martino, J. J, Koganti, A, et al. Melanoma mouse model implicates metabotropic glutamate signaling in melanocytic neoplasia. Nat Genet (2003). May; 34(1), 108-12.

[43] Ohtani, Y, Harada, T, Funasaka, Y, Nakao, K, Takahara, C, Abdel-daim, M, et al. Metabotropic glutamate receptor subtype-1 is essential for in vivo growth of melanoma. Oncogene (2008). Sep 8; 27, 7162-70.

[44] Namkoong, J, Shin, S. S, Lee, H. J, Marin, Y. E, Wall, B. A, Goydos, J. S, et al. Metabotropic glutamate receptor 1 and glutamate signaling in human melanoma. Cancer Res (2007). Mar $1 ;$, 67(5), 2298-305.

[45] Shin, S. S, Namkoong, J, Wall, B. A, Gleason, R, Lee, H. J, \& Chen, S. Oncogenic activities of metabotropic glutamate receptor $1(\mathrm{Grm} 1)$ in melanocyte transformation. Pigment cell \& melanoma research (2008). Jun;, 21(3), 368-78.

[46] Marin, Y. E, Namkoong, J, Cohen-solal, K, Shin, S. S, Martino, J. J, Oka, M, et al. Stimulation of oncogenic metabotropic glutamate receptor 1 in melanoma cells activates ERK1/2 via PKCepsilon. Cell Signal (2006). Aug;, 18(8), 1279-86.

[47] Marin, Y. E, \& Chen, S. Involvement of metabotropic glutamate receptor 1, a G protein coupled receptor, in melanoma development. J Mol Med (2004). Nov;, 82(11), 735-49.

[48] Busca, R, Abbe, P, Mantoux, F, Aberdam, E, Peyssonnaux, C, Eychene, A, et al. Ras mediates the cAMP-dependent activation of extracellular signal-regulated kinases (ERKs) in melanocytes. Embo J (2000). Jun 15;, 19(12), 2900-10.

[49] Ferraguti, F, Baldani-guerra, B, Corsi, M, Nakanishi, S, \& Corti, C. Activation of the extracellular signal-regulated kinase 2 by metabotropic glutamate receptors. Eur J Neurosci (1999). Jun;, 11(6), 2073-82.

[50] Thandi, S, Blank, J. L, \& Challiss, R. A. Group-I metabotropic glutamate receptors, mGlu1a and mGlu5a, couple to extracellular signal-regulated kinase (ERK) activation via distinct, but overlapping, signalling pathways. J Neurochem (2002). Dec;" 83(5), 1139-53. 
[51] Choe, E. S, \& Wang, J. Q. Regulation of transcription factor phosphorylation by metabotropic glutamate receptor-associated signaling pathways in rat striatal neurons. Neuroscience (2002). , 114(3), 557-65.

[52] Stepulak, A, Luksch, H, Gebhardt, C, Uckermann, O, Marzahn, J, Sifringer, M, et al. Expression of glutamate receptor subunits in human cancers. Histochem Cell Biol (2009). Oct;, 132(4), 435-45.

[53] Onken, M. D, Worley, L. A, Long, M. D, Duan, S, Council, M. L, Bowcock, A. M, et al. Oncogenic mutations in GNAQ occur early in uveal melanoma. Invest Ophthalmol Vis Sci (2008). Dec;

[54] Degenhardt, K, \& White, E. A mouse model system to genetically dissect the molecular mechanisms regulating tumorigenesis. Clin Cancer Res (2006). Sep 15;, 12(18), 5298-304.

[55] Martino, J. J, Wall, B. A, Mastrantoni, E, \& Wilimczyk, B. J. La Cava SN, Degenhardt $\mathrm{K}$, et al. Metabotropic glutamate receptor 1 (Grm1) is an oncogene in epithilial cells. Oncogene (2012). Accepted, September 2012.

[56] Chang, H. J, Yoo, B. C, Lim, S. B, Jeong, S. Y, Kim, W. H, \& Park, J. G. Metabotropic glutamate receptor 4 expression in colorectal carcinoma and its prognostic significance. Clin Cancer Res (2005). May 1; 11(9), 3288-95.

[57] Choi, K. Y, Chang, K, Pickel, J. M, \& Badger, J. D. nd, Roche KW. Expression of the metabotropic glutamate receptor 5 (mGluR5) induces melanoma in transgenic mice. Proc Natl Acad Sci U S A (2011). Sep 13;, 108(37), 15219-24.

[58] Wyllie, A. H, Carder, P. J, Clarke, A. R, Cripps, K. J, Gledhill, S, Greaves, M. F, et al. Apoptosis in carcinogenesis: the role of Cold Spring Harb Symp Quant Biol (1994). , 53.

[59] Wyllie, A. H, Kerr, J. F, \& Currie, A. R. Cell death: the significance of apoptosis. Int Rev Cytol (1980). , 68, 251-306.

[60] Thompson, C. B. Apoptosis in the pathogenesis and treatment of disease. Science (1995). Mar 10;, 267(5203), 1456-62.

[61] Engelman, J. A, Luo, J, \& Cantley, L. C. The evolution of phosphatidylinositol 3-kinases as regulators of growth and metabolism. Nat Rev Genet (2006). Aug;, 7(8), 606-19.

[62] Cantley, L. C. The phosphoinositide 3-kinase pathway. Science (2002). May 31; 296(5573), 1655-7.

[63] Hu, P, Margolis, B, Skolnik, E. Y, Lammers, R, Ullrich, A, \& Schlessinger, J. Interaction of phosphatidylinositol 3-kinase-associated with epidermal growth factor and platelet-derived growth factor receptors. Mol Cell Biol (1992). Mar;12(3):981-90., 85.

[64] Rodriguez-viciana, P, Marte, B. M, Warne, P. H, \& Downward, J. Phosphatidylinositol 3' kinase: one of the effectors of Ras. Philos Trans R Soc Lond B Biol Sci (1996). Feb 29; discussion 31-2., 351(1336), 225-31. 
[65] Jiang, B. H, \& Liu, L. Z. PI3K/PTEN signaling in tumorigenesis and angiogenesis. Biochim Biophys Acta (2008). Jan;, 1784(1), 150-8.

[66] Du, K, \& Montminy, M. CREB is a regulatory target for the protein kinase Akt/PKB. J Biol Chem (1998). Dec 4; 273(49), 32377-9.

[67] Zhou, H, Li, X. M, Meinkoth, J, \& Pittman, R. N. Akt regulates cell survival and apoptosis at a postmitochondrial level. J Cell Biol (2000). Oct 30;, 151(3), 483-94.

[68] Romashkova, J. A, Makarov, S. S, \& Is, N. F-k. a. p. p. a. B. a target of AKT in antiapoptotic PDGF signalling. Nature (1999). Sep 2;, 401(6748), 86-90.

[69] Kane, L. P, Shapiro, V. S, Stokoe, D, \& Weiss, A. Induction of NF-kappaB by the Akt/PKB kinase. Curr Biol (1999). Jun 3; 9(11), 601-4.

[70] Mayo, L. D, \& Donner, D. B. A phosphatidylinositol 3-kinase/Akt pathway promotes translocation of Mdm2 from the cytoplasm to the nucleus. Proc Natl Acad Sci U S A (2001). Sep 25; 98(20), 11598-603.

[71] Zhou, B. P, Liao, Y, Xia, W, Zou, Y, Spohn, B, \& Hung, M. C. HER-2/neu induces ubiquitination via Akt-mediated MDM2 phosphorylation. Nat Cell Biol (2001). Nov; 3(11):973-82., 53.

[72] Staal, S. P. Molecular cloning of the akt oncogene and its human homologues AKT1 and AKT2: amplification of AKT1 in a primary human gastric adenocarcinoma. Proc Natl Acad Sci U S A (1987). Jul;, 84(14), 5034-7.

[73] Bellacosa, A, De Feo, D, Godwin, A. K, Bell, D. W, Cheng, J. Q, Altomare, D. A, et al. Molecular alterations of the AKT2 oncogene in ovarian and breast carcinomas. Int $J$ Cancer (1995). Aug 22; 64(4), 280-5.

[74] Cheng, J. Q, Ruggeri, B, Klein, W. M, Sonoda, G, Altomare, D. A, Watson, D. K, et al. Amplification of AKT2 in human pancreatic cells and inhibition of AKT2 expression and tumorigenicity by antisense RNA. Proc Natl Acad Sci U S A (1996). Apr 16;, 93(8), 3636-41.

[75] Schwartz, P. S, Manion, M. K, Emerson, C. B, Fry, J. S, Schulz, C. M, Sweet, I. R, et al. Methoxy antimycin reveals a unique mechanism for Bcl-x (L) inhibition. Mol Cancer Ther (2007). Jul;, 6(7), 2073-80.

[76] Okumura, N, Yoshida, H, Kitagishi, Y, Murakami, M, Nishimura, Y, Matsuda, S, \& Akt, P. I3K. PTEN Signaling as a Molecular Target in Leukemia Angiogenesis. Adv Hematol (2012).

[77] Maehama, T, \& Dixon, J. E. The tumor suppressor, PTEN/MMAC1, dephosphorylates the lipid second messenger, phosphatidylinositol 3,4,5-trisphosphate. J Biol Chem (1998). May 29;, 273(22), 13375-8.

[78] Huntington, J. T, \& Shields, J. M. Der CJ, Wyatt CA, Benbow U, Slingluff CL, Jr., et al. Overexpression of collagenase 1 (MMP-1) is mediated by the ERK pathway in inva- 
sive melanoma cells: role of BRAF mutation and fibroblast growth factor signaling. $J$ Biol Chem (2004). Aug 6;, 279(32), 33168-76.

[79] Satyamoorthy, K, Li, G, Gerrero, M. R, Brose, M. S, Volpe, P, Weber, B. L, et al. Constitutive mitogen-activated protein kinase activation in melanoma is mediated by both BRAF mutations and autocrine growth factor stimulation. Cancer Res (2003). Feb $15 ;$; 63(4), 756-9.

[80] Clark, B. P, Baker, S. R, Goldsworthy, J, Harris, J. R, \& Kingston, A. E. methyl-4-carboxyphenylglycine (LY367385) selectively antagonises metabotropic glutamate mGluR1 receptors. Bioorg Med Chem Lett (1997). Nov 4; 7(21), 2777-80.

[81] Carroll, F. Y, Stolle, A, Beart, P. M, Voerste, A, Brabet, I, Mauler, F, et al. BAY36-7620: a potent non-competitive mGlu1 receptor antagonist with inverse agonist activity. Mol Pharmacol (2001). May;, 59(5), 965-73.

[82] Francesconi, A, \& Duvoisin, R. Role of the second and third intracellular loops of metabotropic glutamate receptors in mediatic dual signal transduction activation. J biol Chem (1998). , 273, 5615-24.

[83] Saez, E, Nelson, M. C, Eshelman, B, Banayo, E, Koder, A, Cho, G. J, et al. Identification of ligands and coligands for the ecdysone-regulated gene switch. Proc Natl Acad Sci U S A (2000). Dec 19;, 97(26), 14512-7.

[84] Wangari-talbot, J, Wall, B. A, Goydos, J. S, \& Chen, S. Functional Effects of GRM1 Suppression in Human Melanoma Cells. Mol Cancer Res (2012). Jul 13.

[85] Marin, Y. E, Namkoong, J, Shin, S. S, Raines, J, Degenhardt, K, White, E, et al. Grm5 expression is not required for the oncogenic role of Grm1 in melanocytes. Neuropharmacology (2005). Suppl , 1, 70-9.

[86] Melendez, R. I, \& Kalivas, P. W. Metabotropic glutamate receptor regulation of extracellular glutamate levels in the prefrontal cortex. Ann N Y Acad Sci (2003). Nov; 1003, 443-4.

[87] Melendez, R. I, Vuthiganon, J, \& Kalivas, P. W. Regulation of extracellular glutamate in the prefrontal cortex: focus on the cystine glutamate exchanger and group I metabotropic glutamate receptors. J Pharmacol Exp Ther (2005). Jul;; 314(1), 139-47.

[88] Van den Bosch LThe causes and mechanism of selective motor neuron death in amyotrophic lateral sclerosis]. Verh K Acad Geneeskd Belg (2006). , 68(4), 249-69.

[89] Van Den Bosch LVan Damme P, Bogaert E, Robberecht W. The role of excitotoxicity in the pathogenesis of amyotrophic lateral sclerosis. Biochim Biophys Acta (2006). NovDec;1762(11-12):1068-82.

[90] Yip, D, Le, M. N, Chan, J. L, Lee, J. H, Mehnert, J. A, Yudd, A, et al. A phase 0 trial of riluzole in patients with resectable stage III and IV melanoma. Clin Cancer Res (2009). Jun $1 ;$, 15(11), 3896-902. 
[91] Davies, H, Bignell, G. R, Cox, C, Stephens, P, Edkins, S, Clegg, S, et al. Mutations of the BRAF gene in human cancer. Nature (2002). Jun 27; 417(6892), 949-54.

[92] Prickett, T. D, Wei, X, Cardenas-navia, I, Teer, J. K, Lin, J. C, Walia, V, et al. Exon capture analysis of $G$ protein-coupled receptors identifies activating mutations in GRM3 in melanoma. Nat Genet (2011). Nov;, 43(11), 1119-26.

[93] Ohtani, Y, Harada, T, Funasaka, Y, Nakao, K, Takahara, C, Abdel-daim, M, et al. Metabotropic glutamate receptor subtype-1 is essential for in vivo growth of melanoma. Oncogene (2008). Dec 4; 27(57), 7162-70.

[94] Li, S, Huang, S, \& Peng, S. B. Overexpression of G protein-coupled receptors in cancer cells: involvement in tumor progression. Int J Oncol (2005). Nov; 27(5), 1329-39. 